TRANSACTIONS OF THE

AMERICAN MATHEMATICAL SOCIETY

Volume 354, Number 12, Pages 4893-4907

S 0002-9947(02)03097-0

Article electronically published on August 1, 2002

\title{
NONISOTROPIC STRONGLY SINGULAR INTEGRAL OPERATORS
}

\author{
BASSAM SHAYYA
}

\begin{abstract}
We consider a class of strongly singular integral operators which include those studied by Wainger, and Fefferman and Stein, and extend the results concerning the $L^{p}$ boundedness of these operators to the nonisotropic setting. We also describe a geometric property of the underlying space which helps us show that our results are sharp.
\end{abstract}

\section{INTRODUCTION}

Let $0<a_{1} \leq a_{2}, \nu=a_{1}+a_{2}$, and consider the one-parameter group $\left\{\delta_{t}\right\}_{t>0}$ of nonisotropic dilations on $\mathbb{R}^{2}$ given by $\delta_{t}:\left(x_{1}, x_{2}\right) \longmapsto\left(t^{a_{1}} x_{1}, t^{a_{2}} x_{2}\right)$. Following Stein and Wainger [9], we define a function $\rho: \mathbb{R}^{2} \rightarrow[0, \infty)$ as follows. If $x \neq$ $0,\left|\delta_{\frac{1}{t}} x\right|$ as a function of $t$ is strictly decreasing and is therefore equal to 1 for a unique value of $t$. Define $\rho(x)$ to be this unique $t$. If $x=0$, set $\rho(x)=0$. Then $\rho$ is continuous, $\rho(x+y) \leq C(\rho(x)+\rho(y))$ for some $C>0$, and $\rho\left(\delta_{t} x\right)=$ $t \rho(x)$ for every $t>0$. This function $\rho$ is often called a $\delta_{t}$-homogeneous distance function. The purpose of this paper is to study the $L^{p}$ boundedness of the singular integral operator defined on the space $C_{0}^{\infty}\left(\mathbb{R}^{2}\right)$ of infinitely differentiable functions of compact support by

$$
T \varphi(x)=\lim _{\epsilon \rightarrow 0} \int_{1 \geq \rho(y) \geq \epsilon} \frac{e^{i / \rho(y)^{\beta}}}{\rho(y)^{\alpha}} \varphi(x-y) d y,
$$

where $\alpha, \beta>0$. Using the generalized system of polar coordinates that one has in this setting, it is easy to see that the function $1 / \rho(y)^{\alpha}$ is integrable near the origin if $\alpha<\nu$. So we assume $\alpha \geq \nu$. Then a straightforward argument of integration by parts shows us that the limit in (1) exists if $\beta>\alpha-\nu$.

In the special case $\rho(y)=|y|\left(a_{1}=a_{2}=1\right)$, and in the setting of $\mathbb{R}^{n}$, it was shown in Wainger 10 that $T$ extends to a bounded operator on $L^{p}\left(\mathbb{R}^{n}\right)$ for $|1 / p-1 / 2|<((n / 2) \beta-\alpha+n) / n \beta$, and that $T$ is not bounded on $L^{p}\left(\mathbb{R}^{n}\right)$ if $|1 / p-1 / 2|>((n / 2) \beta-\alpha+n) / n \beta$. This was obtained by fully describing the asymptotic behavior near $\infty$ of the Fourier transform of the kernel of $T$. The question of whether or not $T$ remains bounded on $L^{p}\left(\mathbb{R}^{n}\right)$ when $|1 / p-1 / 2|=$ $((n / 2) \beta-\alpha+n) / n \beta(\alpha>n)$ was answered positively in Fefferman and Stein [3] using complex interpolation on Hardy spaces after proving the following theorem:

Received by the editors May 6, 1997 .

2000 Mathematics Subject Classification. Primary 42B20; Secondary 42B15.

(C)2002 American Mathematical Society 
Theorem A. Let $L$ be an integrable function on $\mathbb{R}^{n}$ with $L(x)=0$ for $|x|>1$. Assume there exists $\theta \in(0,1)$ such that

$$
\int_{|x|>2|y|^{1-\theta}}|L(x-y)-L(x)| d x \leq B
$$

for $|y|<1$, and

$$
|\widehat{L}(\xi)| \leq \frac{B}{(1+|\xi|)^{n \theta / 2}} .
$$

Then the transformation $S(f)=L * f$ is bounded from $H^{1}\left(\mathbb{R}^{n}\right)$ to $L^{1}\left(\mathbb{R}^{n}\right)$ with a bound that depends on $\theta$ and $B$ but not on the $L^{1}$ norm of $L$.

The function defined by $L_{\epsilon}(x)=e^{i /|x|^{\beta}} /|x|^{n}$ for $\epsilon \leq|x| \leq 1$, and $L_{\epsilon}(x)=$ 0 otherwise, satisfies the hypothesis of Theorem $\mathrm{A}$ with $\theta=\beta /(\beta+1)$ and $B$ independent of $\epsilon$ (see [2], 3], and [10]). For further results in the radial case, we refer the reader to 4 , [5], and [6].

We are going to extend the above results to the nonisotropic setting. To extend Theorem $\mathrm{A}$ we introduce another distance function $\rho_{\beta}$ which will better describe the smoothness of the kernel of a nonisotropic strongly singular integral operator and the decay of its Fourier transform. It will turn out that the balls associated to $\rho$, and those associated to $\rho_{\beta}$, are related by a geometric property which will play an important role in studying the operator $T$. Our main results on the $L^{p}$ boundedness of $T$ are stated in the following theorem.

Theorem 1. Suppose $\beta>\alpha-\nu \geq 0$. For $\varphi \in C_{0}^{\infty}$, define

$$
T \varphi(x)=\lim _{\epsilon \rightarrow 0} \int_{1 \geq \rho(y) \geq \epsilon} \frac{e^{i / \rho(y)^{\beta}}}{\rho(y)^{\alpha}} \varphi(x-y) d y .
$$

Then:

(i) If $\alpha>\nu$, then $T$ extends to a bounded linear operator on $L^{p}\left(\mathbb{R}^{2}\right)$ for

$$
\left|\frac{1}{p}-\frac{1}{2}\right| \leq \frac{\beta-\alpha+\nu}{2 \beta} .
$$

If $\alpha=\nu$, then $T$ is bounded on $L^{p}\left(\mathbb{R}^{2}\right)$ for $1<p<\infty$. On the other hand,

(ii) if

$$
\left|\frac{1}{p}-\frac{1}{2}\right|>\frac{\beta-\alpha+\nu}{2 \beta},
$$

then $T$ is not bounded on $L^{p}\left(\mathbb{R}^{2}\right)$.

If $x_{0} \in \mathbb{R}^{2}$, and $r \geq 0$, we define a $\rho$-ball by $B\left(x_{0}, r\right)=\left\{x \in \mathbb{R}^{2}: \rho\left(x-x_{0}\right) \leq r\right\}$. A 1 -atom is a function $a \in L^{\infty}\left(\mathbb{R}^{2}\right)$ supported in a $\rho$-ball $B\left(x_{0}, r\right)$ such that

(i) $\|a\|_{L^{\infty}} \leq r^{-\nu}$, and

(ii) $\int a(x) d x=0$.

Following Coifman and Weiss [1], we define $H_{\rho}^{1}\left(\mathbb{R}^{2}\right)$ as the set of all $f \in S^{\prime}$ that can be represented in the form $f=\sum_{i=0}^{\infty} \mu_{i} a_{i}$, where each $a_{i}$ is a 1 -atom and $\sum_{i=0}^{\infty}\left|\mu_{i}\right|<\infty$. Also, for $f \in H_{\rho}^{1}\left(\mathbb{R}^{2}\right)$ we have $\|f\|_{H_{\rho}^{1}}=\inf \left\{\sum\left|\mu_{i}\right|: f=\sum \mu_{i} a_{i}\right\}$. Throughout this paper a constant is a positive real number that depends only on $\alpha, \beta, a_{1}$, and $a_{2}$. $c$ will always denote a constant which does not necessarily have the same value every time it appears. 


\section{ACKNOWLEDGEMENTS}

This paper is based in part on my Ph.D. thesis written at the University of Wisconsin-Madison, and I would like to take this opportunity to thank my teacher and advisor, S. Wainger, for his support and valuable guidance. I would also like to thank A. Seeger for many helpful comments and suggestions concerning the content of this paper.

\section{The $L^{p}$ Inequality}

We start this section by stating some further properties of the function $\rho$.

Proposition 1. (i) $\rho(x)$ is infinitely differentiable in $\mathbb{R}^{2}-0$. Also, for $x \neq 0$,

$$
\left|\frac{\partial \rho}{\partial x_{1}}(x)\right| \leq C \rho(x)^{1-a_{1}} \text { and }\left|\frac{\partial \rho}{\partial x_{2}}(x)\right| \leq C \rho(x)^{1-a_{2}}
$$

for some $C>0$.

(ii) If $|x| \geq 1$, then $\rho(x)^{a_{1}} \leq|x| \leq \rho(x)^{a_{2}}$.

(iii) If $|x| \leq 1$, then $\rho(x)^{a_{1}} \geq|x| \geq \rho(x)^{a_{2}}$.

(iv) If $f \in L^{1}\left(\mathbb{R}^{2}\right)$ or $f \geq 0$, then

$$
\int_{\mathbb{R}^{2}} f(x) d x=\int_{0}^{2 \pi} \Omega(\theta)\left[\int_{0}^{\infty} f\left(\delta_{r}(\cos \theta, \sin \theta)\right) r^{\nu-1} d r\right] d \theta
$$

where $\Omega(\theta)=a_{1}+\left(a_{2}-a_{1}\right) \sin ^{2} \theta$.

Part (iv) describes the generalized polar coordinates mentioned above. For a proof of Proposition 1] see [9].

For $\beta>0$ we associate to $\rho$ a function $\rho_{\beta}$ as follows. For $t>0$ and $x \in \mathbb{R}^{2}$, define

$$
\gamma_{t}(x)=t^{\beta} \delta_{t}(y)=\left(t^{a_{1}+\beta} x_{1}, t^{a_{2}+\beta} x_{2}\right),
$$

and let $\rho_{\beta}$ be the distance function corresponding to the group $\left\{\gamma_{t}\right\}_{t>0}$. The geometric property, mentioned before, that relates $\rho_{\beta}$-balls to $\rho$-balls will be described in detail in the next section. For now let us note that

$$
\rho(x) \leq \rho_{\beta}(x) \text {, if } \rho(x) \leq 1 .
$$

We start by proving the following generalization of Theorem $\mathrm{A}$.

Theorem 2. Let $K_{0} \in L^{1}\left(\mathbb{R}^{2}\right)$ with $K_{0}(x)=0$ for $\rho(x)>1$. Assume there exist $\beta>0$ and a constant $C$ such that

$$
\int_{\rho(x)>C \rho_{\beta}(y)}\left|K_{0}(x-y)-K_{0}(x)\right| d x \leq B_{0}
$$

for $\rho(y)<1$, and

$$
\left|\widehat{K_{0}}(\xi)\right| \leq \frac{B_{0}}{\left(1+\rho_{\beta}(\xi)\right)^{\beta}} .
$$

Then the transformation $T_{0}(f)=K_{0} * f$ is bounded from $H_{\rho_{\beta}}^{1}\left(\mathbb{R}^{2}\right)$ to $L^{1}\left(\mathbb{R}^{2}\right)$ with a bound that depends on $\beta, B_{0}$ and $C$ but not on the $L^{1}$ norm of $K_{0}$. 
Proof. It suffices to show that $\left\|T_{0}(a)\right\|_{L^{1}} \leq c$ for each 1-atom $a$, with $c$ independent of the $L^{1}$ norm of $K_{0}$ and the choice of $a$. Let $a$ be a 1-atom supported in a $\rho_{\beta}$-ball $B=B\left(x_{0}, r\right)$. Since $T_{0}$ is translation invariant, we can take $x_{0}=0$. Then $T_{0}(a)$ is supported in a $\rho_{\beta}$-ball $B(0, c(1+r))$. By part (iv) of Proposition 1 , the Lebesgue measure $|B(0, c(1+r))|$ of $B(0, c(1+r))$ is $\leq c(1+r)^{2 \beta+\nu}$. So if $r \geq 1$,

$$
\begin{aligned}
\left\|T_{0}(a)\right\|_{L^{1}} & \leq c(1+r)^{\beta+\nu / 2}\left\|T_{0}(a)\right\|_{L^{2}} \\
& \leq c(1+r)^{\beta+\nu / 2}\|a\|_{L^{2}} \\
& \leq c(1+r)^{\beta+\nu / 2} \frac{1}{r^{\beta+\nu / 2}} \\
& \leq c .
\end{aligned}
$$

Suppose $r<1$ and consider the $\rho$-ball $B^{*}=B(0, C r)$. Then

$$
\begin{aligned}
\left\|T_{0}(a)\right\|_{L^{1}\left(\mathbb{R}^{2}-B^{*}\right)} & =\int_{\mathbb{R}^{2}-B^{*}}\left|\int K_{0}(x-y) a(y) d y\right| d x \\
& =\int_{\mathbb{R}^{2}-B^{*}}\left|\int\left(K_{0}(x-y)-K_{0}(x)\right) a(y) d y\right| d x \\
& \leq \int|a(y)| \int_{\mathbb{R}^{2}-B^{*}}\left|K_{0}(x-y)-K_{0}(x)\right| d x d y \\
& \leq \int|a(y)| \int_{\rho(x)>C \rho_{\beta}(y)}\left|K_{0}(x-y)-K_{0}(x)\right| d x d y \\
& \leq B_{0}\|a\|_{L^{1}} \\
& \leq c
\end{aligned}
$$

and

$$
\begin{aligned}
\left\|T_{0}(a)\right\|_{L^{1}\left(B^{*}\right) \leq}^{2} \leq & \left|B^{*}\right|\left\|T_{0}(a)\right\|_{L^{2}}^{2} \\
\leq & c r^{\nu}\left\|\widehat{T_{0}(a)}\right\|_{L^{2}}^{2} \\
= & c r^{\nu} \int\left|\widehat{K_{0}}(\xi)\right|^{2}|\widehat{a}(\xi)|^{2} d \xi \\
= & c r^{\nu} \int_{\rho_{\beta}(\xi) \geq 1 / r}\left|\widehat{K_{0}}(\xi)\right|^{2}|\widehat{a}(\xi)|^{2} d \xi \\
& +c r^{\nu} \int_{\rho_{\beta}(\xi) \leq 1 / r}\left|\widehat{K_{0}}(\xi)\right|^{2}|\widehat{a}(\xi)|^{2} d \xi \\
\leq & c r^{\nu} \int_{\rho_{\beta}(\xi) \geq 1 / r} \rho_{\beta}(\xi)^{-2 \beta}|\widehat{a}(\xi)|^{2} d \xi \\
& +c r^{\nu}\|\widehat{a}\|_{L^{\infty}}^{2} \int_{\rho_{\beta}(\xi) \leq 1 / r} \rho_{\beta}(\xi)^{-2 \beta} d \xi \\
\leq & c r^{\nu+2 \beta} \int_{\rho_{\beta}(\xi) \geq 1 / r}|\widehat{a}(\xi)|^{2} d \xi \\
& +c r^{\nu}\|a\|_{L^{1}}^{2} \int_{0}^{1 / r} s^{-2 \beta} s^{\nu+2 \beta-1} d s \\
\leq & c r^{\nu+2 \beta}\|a\|_{L^{2}}^{2}+c r^{\nu} \int_{0}^{1 / r} s^{\nu-1} d s \\
\leq & c .
\end{aligned}
$$


Hence $\left\|T_{0}(a)\right\|_{L^{1}}=\left\|T_{0}(a)\right\|_{L^{1}\left(B^{*}\right)}+\left\|T_{0}(a)\right\|_{L^{1}\left(\mathbb{R}^{2}-B^{*}\right)} \leq c$. This completes the proof.

For $y \neq 0$ define $K(y)=e^{i / \rho(y)^{\beta}} / \rho(y)^{\alpha}$, and set

$$
K_{\epsilon}(y)= \begin{cases}K(y) & \text { if } \epsilon \leq \rho(y) \leq 1 \\ 0 & \text { otherwise }\end{cases}
$$

$(0<\epsilon \leq 1)$. Now for $f \in L^{p}\left(\mathbb{R}^{2}\right), 1 \leq p \leq \infty$, define $T_{\epsilon} f=K_{\epsilon} * f$. Then if $\beta>\alpha-\nu \geq 0$ and $\varphi \in C_{0}^{\infty}\left(\mathbb{R}^{2}\right)$, it follows that $T \varphi(x)=\lim _{\epsilon \rightarrow 0} T_{\epsilon} \varphi(x)$ for every $x \in \mathbb{R}^{2}$.

Theorem 3. Suppose $\beta>0$ and $\beta \geq \alpha-\nu \geq 0$. If $|1 / p-1 / 2| \leq(\beta-\alpha+\nu) /(2 \beta)$ $(\alpha>\nu)$ or $1<p<\infty(\alpha=\nu)$, we have

$$
\left\|T_{\epsilon} f\right\|_{L^{p}} \leq A_{p}\|f\|_{L^{p}}
$$

for every $f \in L^{p}$. The constant $A_{p}$ is independent of $\epsilon$.

A standard limiting argument shows that part (i) of Theorem 1 is an immediate consequence of Theorem [3. Part (i) of Proposition 1 tells us that

$$
\left|\frac{\partial K_{\epsilon}}{\partial x_{1}}(x)\right| \leq c \rho(x)^{-\alpha-\beta-a_{1}} \text { and }\left|\frac{\partial K_{\epsilon}}{\partial x_{2}}(x)\right| \leq c \rho(x)^{-\alpha-\beta-a_{2}} .
$$

So, if $\alpha=\nu$, it can be easily checked that

$$
\int_{\rho(x)>C \rho_{\beta}(y)}\left|K_{\epsilon}(x-y)-K_{\epsilon}(x)\right| d x \leq B_{0},
$$

uniformly in $\epsilon$. In the next theorem, we estimate the Fourier transform of $K_{\epsilon}$, and it will turn out that if $\alpha=\nu$, then $\left|\widehat{K}_{\epsilon}(\xi)\right| \leq B_{0}\left(1+\rho_{\beta}(\xi)\right)^{-\beta}$. Theorem 2 then tells us that $T_{\epsilon}$ is bounded from $H_{\rho_{\beta}}^{1}\left(\mathbb{R}^{2}\right)$ to $L^{1}\left(\mathbb{R}^{2}\right)$ with a bound that is independent of $\epsilon$. So our next task is to estimate $\widehat{K}_{\epsilon}$, and for this we need the following lemma of van der Corput, which can be found in [8, pages 332-334].

Proposition 2. Suppose $\phi$ is real-valued and smooth in $(a, b)$, and that $\left|\phi^{(k)}(x)\right| \geq$ $\lambda>0$ for all $x \in(a, b)$. Then

$$
\left|\int_{a}^{b} e^{i \phi(x)} d x\right| \leq c_{k} \lambda^{-1 / k}
$$

holds when:

(i) $k \geq 2$, or

(ii) $k=1$ and $\phi^{\prime \prime}(x)$ has at most one zero. Also, $c_{k}=5\left(2^{k}\right)-4$.

Now if $0<a<b, \phi$ and $\psi$ are real-valued and smooth in $(a, b)$, and $\left|\phi^{(k)}(x)\right| \geq$ $\lambda / x^{s}(s \geq 0)$ (when $k=1$ we also assume that $\phi^{\prime \prime}(x)$ has at most one zero), then

$$
\int_{a}^{b} e^{i \phi(x)} \psi(x) d x=\int_{a}^{b} \psi(x) F^{\prime}(x) d x
$$

where $F(x)=\int_{a}^{x} e^{i \phi(t)} d t$. By Proposition 2, $|F(x)| \leq c_{k} \lambda^{-1 / k} x^{s / k}$ for $x \in[a, b]$, and on integrating the above integral by parts it follows that

$$
\left|\int_{a}^{b} e^{i \phi(x)} \psi(x) d x\right| \leq c_{k} \lambda^{-1 / k}\left[b^{s / k}|\psi(b)|+\int_{a}^{b} x^{s / k}\left|\psi^{\prime}(x)\right| d x\right] .
$$


In particular, if $s=0$, then

$$
\left|\int_{a}^{b} e^{i \phi(x)} \psi(x) d x\right| \leq c_{k} \lambda^{-1 / k}\left[|\psi(b)|+\int_{a}^{b}\left|\psi^{\prime}(x)\right| d x\right] .
$$

Theorem 4. Suppose $\beta>0$ and $\beta \geq \alpha-\nu \geq 0$. Then

$$
\left|\widehat{\left(\frac{K_{\epsilon}}{\rho(.)^{i v}}\right)(\xi)}\right| \leq B \frac{1+|v|}{\left(1+\rho_{\beta}(\xi)\right)^{\beta-\alpha+\nu}},
$$

$-\infty<v<+\infty$. The constant $B$ is independent of $\epsilon$.

Proof. If $\rho^{\prime}$ is the distance function corresponding to the group $\left\{\delta_{t}^{\prime}\right\}_{t>0}$, where $\delta_{t}^{\prime} x=\left(t x_{1}, t^{a_{2} / a_{1}} x_{2}\right)$, then it is not hard to see that $\rho(y)=\rho^{\prime}(y)^{1 / a_{1}}$ and $\rho_{\beta}(y)=$ $\rho_{\beta / a_{1}}^{\prime}(y)^{1 / a_{1}}$ for every $y \in \mathbb{R}^{2}$. Therefore, we can assume $a_{1}=1$ (then $\nu=1+a_{2} \geq$ $2)$. If $\rho_{\beta}(\xi)$ is small, an easy argument of integration by parts shows that the Fourier transform of $K_{\epsilon} / \rho(.)^{i v}$ is bounded. So it suffices to prove the theorem for large values of $\rho_{\beta}(\xi)$. Furthermore, since $\rho\left(x_{1}, x_{2}\right)=\rho\left(-x_{1}, x_{2}\right)=\rho\left(-x_{1},-x_{2}\right)$, it is enough to look at $\xi=\left(\xi_{1}, \xi_{2}\right)$ with $\xi_{1}, \xi_{2} \geq 0$. Write

$$
\left(\widehat{\frac{K_{\epsilon}}{\rho(.)^{i v}}}\right)(\xi)=I_{1}+I_{2}
$$

where

$$
I_{1}=\int_{\rho_{\beta}(x) \leq C_{0} \lambda(\xi)} \frac{K_{\epsilon}(x)}{\rho(x)^{i v}} e^{i \xi \cdot x} d x
$$

and

$$
I_{2}=\int_{\rho_{\beta}(x) \geq C_{0} \lambda(\xi)} \frac{K_{\epsilon}(x)}{\rho(x)^{i v}} e^{i \xi \cdot x} d x
$$

$C_{0}$ and $\lambda(\xi)$ are going to be chosen. For $r>0$, set $f(r)=\frac{d}{d r}\left|\delta_{r} \xi\right|$. Then $f^{\prime}(r)>0$, and it follows that the equation $\beta r^{-\beta-1}=f(r)$ has a unique solution in $(0, \infty)$. Define $\lambda(\xi)$ to be this unique solution. An easy computation then shows that $\lambda\left(\gamma_{t} \xi\right)=(1 / t) \lambda(\xi)$ for $t>0$, and that there exist constants $C_{1}$ and $C_{2}$ such that $0<C_{1} \leq \lambda(\xi) \leq C_{2}$ whenever $|\xi|=1$. So, writing $\xi=\gamma_{\rho_{\beta}(\xi)} \xi^{\prime}$ with $\left|\xi^{\prime}\right|=1$, we conclude that

$$
\frac{C_{1}}{\rho_{\beta}(\xi)} \leq \lambda(\xi) \leq \frac{C_{2}}{\rho_{\beta}(\xi)}
$$

In generalized polar coordinates,

$$
I_{1}=\int_{0}^{2 \pi} \Omega(\theta)\left[\int_{\epsilon}^{C_{0} \lambda(\xi)} \frac{e^{-i v \ln r}}{r^{\alpha-\nu+1}} e^{i / r^{\beta}} e^{i \xi \cdot \delta_{r}(\cos \theta, \sin \theta)} d r\right] d \theta
$$


Writing $e^{i / r^{\beta}}=\frac{i}{\beta}\left(e^{i / r^{\beta}}\right)^{\prime} r^{\beta+1}$ and integrating the inner integral by parts, it follows that

$$
\begin{aligned}
\left|I_{1}\right| \leq & c \lambda(\xi)^{\beta-\alpha+\nu}+c(1+|v|) \int_{0}^{2 \pi}\left|\int_{\epsilon}^{C_{0} \lambda(\xi)} e^{-i v \ln r} r^{\beta-\alpha+\nu-1} r^{i \Phi_{\theta}(r)} d r\right| d \theta \\
& +c\left|\xi_{1}\right| \int_{0}^{2 \pi}\left|\int_{\epsilon}^{C_{0} \lambda(\xi)} e^{-i v \ln r} r^{\beta-\alpha+\nu} r^{i \Phi_{\theta}(r)} d r\right| d \theta \\
& +c\left|\xi_{2}\right| \int_{0}^{2 \pi}\left|\int_{\epsilon}^{C_{0} \lambda(\xi)} e^{-i v \ln r} r^{\beta-\alpha+2 \nu-2} r^{i \Phi_{\theta}(r)} d r\right| d \theta
\end{aligned}
$$

where $\Phi_{\theta}(r)=r^{-\beta}+r \xi_{1} \cos \theta+r^{\nu-1} \xi_{2} \sin \theta$. Since $\left|\xi_{1}\right| \leq \rho_{\beta}(\xi)^{\beta+1}$ and $\left|\xi_{2}\right| \leq$ $\rho_{\beta}(\xi)^{\beta+\nu-1}$, it follows by (6) that we can find a constant $C_{0}$ small enough that $\left|\Phi_{\theta}^{\prime}(r)\right| \geq \beta / 2 r^{\beta+1}$ for $r \in\left(0, C_{0} \lambda(\xi)\right]$ (uniformly in $\theta$ ). Applying (41) to each of the integrals on the right-hand side of the above inequality, we get

$$
\left|I_{1}\right| \leq c(1+|v|) \lambda(\xi)^{\beta-\alpha+\nu} .
$$

Estimating $I_{2}$ takes more work. As we did for $I_{1}$, we start by expressing the integral in polar coordinates:

$$
I_{2}=\int_{C_{0} \lambda(\xi)}^{1} \frac{e^{-i v \ln r}}{r^{\alpha-\nu+1}} e^{i / r^{\beta}}\left[\int_{0}^{2 \pi} \Omega(\theta) e^{i \xi \cdot \delta_{r}(\cos \theta, \sin \theta)} d \theta\right] d r .
$$

Now using the observation that $\xi \cdot \delta_{r}(\cos \theta, \sin \theta)=\left|\delta_{r} \xi\right| \cos (\theta-h(r))$, where $h(r)=$ $\arctan \left(r^{\nu-2} \xi_{2} / \xi_{1}\right)$, we get

$$
I_{2}=\int_{C_{0} \lambda(\xi)}^{1} \frac{e^{-i v \ln r}}{r^{\alpha-\nu+1}} e^{i / r^{\beta}}\left[\int_{0}^{2 \pi} \Omega(\theta+h(r)) e^{i\left|\delta_{r} \xi\right| \cos \theta} d \theta\right] d r .
$$

Note that $h^{\prime}(r) \leq c / r$. By the method of stationary phase (as stated in [8] page $334])$,

$$
\begin{aligned}
\int_{0}^{2 \pi} & \Omega(\theta+h(r)) e^{i\left|\delta_{r} \xi\right| \cos \theta} d \theta \\
& =\omega_{1} \frac{\Omega(h(r))}{\left|\delta_{r} \xi\right|^{1 / 2}} e^{i\left|\delta_{r} \xi\right|}+\omega_{2} \frac{\Omega(h(r))}{\left|\delta_{r} \xi\right|^{1 / 2}} e^{-i\left|\delta_{r} \xi\right|}+O\left(\left|\delta_{r} \xi\right|^{-3 / 2}\right)
\end{aligned}
$$

$\left(\omega_{1}=\sqrt{2 \pi} e^{-i \pi / 4}\right.$ and $\left.\omega_{2}=\sqrt{2 \pi} e^{i \pi / 4}\right)$. The bounds occurring in the error term in the above equation are independent of $r$ because all derivatives of $\Omega(\theta+h(r))$ with respect to $\theta$ are bounded uniformly in $r$. Let $\psi(r)=e^{-i v \ln r} \Omega(h(r)) /\left|\delta_{r} \xi\right|^{1 / 2} r^{\alpha-\nu+1}$ and $\phi_{\theta}(r)=r^{-\beta}+\left|\delta_{r} \xi\right| \cos \theta$. Then

$$
I_{2}=\omega_{1} \int_{C_{0} \lambda(\xi)}^{1} \psi(r) e^{i \phi_{0}(r)} d r+\omega_{2} \int_{C_{0} \lambda(\xi)}^{1} \psi(r) e^{i \phi_{\pi}(r)} d r+E,
$$

with $|E| \leq c \int_{C_{0} \lambda(\xi)}^{1}\left|\delta_{r} \xi\right|^{-3 / 2} r^{-\alpha+\nu-1} d r$. Now, using the definition of $\lambda(\xi)$, one can easily see that

$$
\frac{1}{\left|\delta_{r} \xi\right|} \leq c \frac{\lambda(\xi)^{\beta+1}}{r}
$$

for $C_{0} \lambda(\xi) \leq r \leq 1$. Therefore,

$$
|E| \leq c \lambda(\xi)^{\frac{3}{2} \beta-\alpha+\nu} .
$$


It remains to estimate

$$
I_{3}=\int_{C_{0} \lambda(\xi)}^{1} \psi(r) e^{i \phi_{0}(r)} d r
$$

and

$$
I_{4}=\int_{C_{0} \lambda(\xi)}^{1} \psi(r) e^{i \phi_{\pi}(r)} d r .
$$

But first let us notice that (8) tells us that if $C_{0} \lambda(\xi) \leq r \leq 1$, then

$$
|\psi(r)| \leq c \frac{\lambda(\xi)^{\frac{\beta}{2}+\frac{1}{2}}}{r^{\alpha-\nu+3 / 2}}
$$

and

$$
\left|\psi^{\prime}(r)\right| \leq c(1+|v|) \frac{\lambda(\xi)^{\frac{\beta}{2}+\frac{1}{2}}}{r^{\alpha-\nu+5 / 2}}
$$

Now $\phi_{\pi}^{\prime}(r)=-\beta r^{-\beta-1}-f(r)$, and since $f(r)>0$, it follows that $\left|\phi_{\pi}^{\prime}(r)\right| \geq$ $c / \lambda(\xi)^{\beta+1}$ for $r \in\left[C_{0} \lambda(\xi), 3 \lambda(\xi) / 2\right]$. Also, for $3 \lambda(\xi) / 2 \leq r \leq 1$,

$$
\left|\phi_{\pi}^{\prime}(r)\right|=\beta r^{-\beta-1}+f(r) \geq f(r) \geq f(\lambda(\xi))=\beta \lambda(\xi)^{-\beta-1} .
$$

Thus $\left|\phi_{\pi}^{\prime}(r)\right| \geq c / \lambda(\xi)^{\beta+1}$ on $\left[C_{0} \lambda(\xi), 1\right]$, and (5) then tells us that

$$
\begin{aligned}
\left|I_{4}\right| & \leq c \lambda(\xi)^{\beta+1}\left[|\psi(1)|+\int_{C_{0} \lambda(\xi)}^{1}\left|\psi^{\prime}(r)\right| d r\right] \\
& \leq c(1+|v|) \lambda(\xi)^{\frac{3}{2} \beta-\alpha+\nu} .
\end{aligned}
$$

For $I_{3}$, we have

$$
I_{3}=\int_{C_{0} \lambda(\xi)}^{3 \lambda(\xi) / 2} \psi(r) e^{i \phi_{0}(r)} d r+\int_{3 \lambda(\xi) / 2}^{1} \psi(r) e^{i \phi_{0}(r)} d r=I_{5}+I_{6} .
$$

On $[3 \lambda(\xi) / 2,1]$,

$$
\begin{aligned}
\phi_{0}^{\prime}(r) & =-\beta r^{-\beta-1}+f(r) \\
& \geq-(2 / 3)^{\beta+1} \beta \lambda(\xi)^{-\beta-1}+f(\lambda(\xi)) \\
& =-(2 / 3)^{\beta+1} \beta \lambda(\xi)^{-\beta-1}+\beta \lambda(\xi)^{-\beta-1} \\
& \geq c \lambda(\xi)^{-\beta-1},
\end{aligned}
$$

and, as before, (5) tells us that

$$
\left|I_{6}\right| \leq c(1+|v|) \lambda(\xi)^{\frac{3}{2} \beta-\alpha+\nu} .
$$

For $C_{0} \lambda(\xi) \leq r \leq 3 \lambda(\xi) / 2$ we have

$$
\phi_{0}^{\prime \prime}(r)=\beta(\beta+1) r^{-\beta-2}+f^{\prime}(r) \geq \beta(\beta+1) r^{-\beta-2} \geq c / \lambda(\xi)^{\beta+2},
$$

and applying (5) one more time, we get

$$
\begin{aligned}
\left|I_{5}\right| & \leq c \lambda(\xi)^{\frac{\beta}{2}+1}\left[|\psi(\lambda(\xi) / 2)|+\int_{C_{0} \lambda(\xi)}^{3 \lambda(\xi) / 2}\left|\psi^{\prime}(r)\right| d r\right] \\
& \leq c(1+|v|) \lambda(\xi)^{\beta-\alpha+\nu} .
\end{aligned}
$$


Combining (7), (9), (10), (12), and (13), we have

$$
\left|\widehat{\left(\frac{K_{\epsilon}}{\rho(.)^{i v}}\right)}(\xi)\right| \leq c(1+|v|) \lambda(\xi)^{\beta-\alpha+\nu}
$$

and by (6),

$$
\left|\widehat{\left(\frac{K_{\epsilon}}{\rho(.)^{i v}}\right)}(\xi)\right| \leq c(1+|v|) \rho_{\beta}(\xi)^{-\beta+\alpha-\nu} .
$$

This completes the proof.

We are now ready to prove Theorem 3 . We use interpolation of analytic families of operators on parabolic Hardy spaces (see [1]).

Proof of Theorem [3. As we mentioned before, if $\alpha=\nu$, then $K_{\epsilon}$ satisfies the hypothesis of Theorem [2] with bounds independent of $\epsilon$, and it follows that $T$ extends to a bounded linear operator on $L^{p}\left(\mathbb{R}^{2}\right)$ for $1<p<\infty$. Assume $\alpha>\nu$. For $z=u+i v \in \mathbb{C}$, set

$$
M_{z}(y)= \begin{cases}\rho(y)^{\beta z-\beta-\nu} e^{i / \rho(y)^{\beta}} & \text { if } \epsilon \leq \rho(y) \leq 1, \\ 0 & \text { otherwise. }\end{cases}
$$

We consider the family $\left\{R_{z}\right\}_{0 \leq u \leq 1}$ of analytic operators defined on the domain of simple functions by

$$
R_{z} f=M_{z} * f .
$$

Clearly, $\frac{R_{\frac{\beta-\alpha+\nu}{\beta}}}{\beta}=T_{\epsilon}$.

If $u=1$, then $\operatorname{Re}[-\beta z+\beta+\nu]=\nu$, and $M_{1+i v}$ satisfies the hypothesis of Theorem 2 with $B_{0}=(1+|v|) B_{1}$ and $B_{1}$ independent of $\epsilon$. Thus

$$
\left\|R_{1+i v} f\right\|_{L^{1}} \leq(1+|v|) A^{\prime}\|f\|_{H_{\rho_{\beta}}^{1}},
$$

and the constant $A^{\prime}$ is independent of $\epsilon$. On the other hand, Theorem 4 tells us that

$$
\left|\widehat{M_{i v}}(\xi)\right| \leq B(1+|v|)
$$

and it follows that

$$
\left\|R_{i v} f\right\|_{L^{2}} \leq(1+|v|) A^{\prime \prime}\|f\|_{L^{2}} .
$$

Now we interpolate between the inequalities in (14) and (15) to conclude that

$$
\left\|R_{u} f\right\|_{L^{p}} \leq A(u, p)\|f\|_{L^{p}}
$$

whenever $0 \leq u<1$ and $\frac{1}{p}=\frac{1-u}{2}+u$. In particular,

$$
\left\|T_{\epsilon} f\right\|_{L^{p}}=\left\|R_{\frac{\beta-\alpha+\nu}{\beta}} f\right\|_{L^{p}} \leq A_{p}\|f\|_{L^{p}}
$$

for $\frac{1}{p}-\frac{1}{2}=\frac{\beta-\alpha+\nu}{2 \beta}$. It follows that

$$
\left\|T_{\epsilon} f\right\|_{L^{p}} \leq A_{p}\|f\|_{L^{p}}
$$

for $0 \leq \frac{1}{p}-\frac{1}{2} \leq \frac{\beta-\alpha+\nu}{2 \beta}$. Finally, a duality argument shows the corresponding result for $2<p<\infty$.

This establishes Theorem 3 and consequently part (i) of Theorem 1 . 


\section{The Sharp Result}

In the last section we showed that, if $\alpha>\nu, T$ extends to a bounded linear operator on $L^{p}$ for $|1 / p-1 / 2| \leq(\beta-\alpha+\nu) / 2 \beta$. In this section we prove that this result is sharp. This was the assertion of part (ii) of Theorem 1 and for convenience, we restate it here as:

Theorem 5. Suppose $T$ extends to a bounded linear operator on $L^{p}, 1 \leq p<\infty$. Then

$$
\left|\frac{1}{p}-\frac{1}{2}\right| \leq \frac{\beta-\alpha+\nu}{2 \beta} .
$$

At this point, outlining the argument that is going to be used in the proof of Theorem 5 will help in understanding some of the details that will follow. We are going to consider an appropriate $\varphi \in C_{0}^{\infty}\left(\mathbb{R}^{2}\right)$ supported in a small neighborhood $U$ of the origin. The goal is, of course, to find a lower bound for $\|T \varphi\|_{L^{p}}$. To achieve this, we examine $|T \varphi(x)|$ at those $x$ 's such that $e^{i / \rho(y)^{\beta}}$ does not oscillate rapidly for $y$ near $x$. For example, suppose that $e^{i / \rho(y)^{\beta}}$ does not oscillate rapidly for $y \in$ $B(0, b)-B(0, a)$, where $0<a<b \leq 1(B(0, a)$ and $B(0, b)$ are $\rho$-balls $)$. For $x \in E \subset$ $B(0, b)-B(0, a)$ let $U_{x}=\left\{y \in \mathbb{R}^{2}: x-y \in U\right\}=$ support of $\varphi$ translated by $x$. To gain the best possible lower bound for $|T \varphi(x)|, U_{x}$ should lie entirely in $B(0, b)-$ $B(0, a)$. Moreover, to gain a satisfactory lower bound for $\|T \varphi\|_{L^{p}}, U_{x}$ should cover most of $B(0, b)-B(0, a)$ as $x$ varies in $E$. For all of this to occur, $\rho_{\beta}(y-x)$, rather than $\rho(y-x)$, should be small for $y \in U_{x}$. This geometric property is the subject of the next lemma.

Lemma 1. Let $0<\epsilon \leq a<b$ and $2 \epsilon^{a_{1}+\beta}<b^{a_{1}}-a^{a_{1}}$. Suppose

$$
\left(a^{a_{1}}+\epsilon^{a_{1}+\beta}\right)^{1 / a_{1}} \leq \rho(x) \leq\left(b^{a_{1}}-\epsilon^{a_{1}+\beta}\right)^{1 / a_{1}}
$$

and $\rho_{\beta}(x-y) \leq \epsilon$. Then $a \leq \rho(y) \leq b$.

Proof. Since $\rho_{\beta}(x-y) \leq \epsilon$, we have $\left|\gamma_{\frac{1}{\epsilon}}(x-y)\right| \leq 1$. It follows that $\left|\delta_{\frac{1}{\epsilon}}(x-y)\right| \leq \epsilon^{\beta}$, and since $a / \epsilon \geq 1$, we get

$$
\epsilon^{\beta} \geq\left|\delta_{\frac{1}{\epsilon}}(x-y)\right|=\left|\delta_{\frac{1}{a} \frac{a}{\epsilon}}(x-y)\right| \geq\left(\frac{a}{\epsilon}\right)^{a_{1}}\left|\delta_{\frac{1}{a}}(x-y)\right|,
$$

or

$$
\left|\delta_{\frac{1}{a}}(x-y)\right| \leq \frac{\epsilon^{a_{1}+\beta}}{a^{a_{1}}} .
$$

Similarly,

$$
\left|\delta_{\frac{1}{b}}(x-y)\right| \leq \frac{\epsilon^{a_{1}+\beta}}{b^{a_{1}}} .
$$

Now, since $\left(a^{a_{1}}+\epsilon^{a_{1}+\beta}\right)^{1 / a_{1}} \leq \rho(x) \leq\left(b^{a_{1}}-\epsilon^{a_{1}+\beta}\right)^{1 / a_{1}}$, we have

$$
\left|\delta \frac{1}{\left(b^{a_{1}}-\epsilon^{a_{1}+\beta}\right)^{1 / a_{1}}} x\right| \leq 1 \leq\left|\delta \frac{1}{\left(a^{a_{1}}+\epsilon^{a_{1}+\beta}\right)^{1 / a_{1}}} x\right| .
$$

The second inequality in (19) tells us that

$$
1 \leq\left|\delta \frac{1}{a\left(1+\frac{\epsilon^{a_{1}+\beta}}{a^{a_{1}}}\right)^{1 / a_{1}}} x\right| \leq \frac{1}{1+\frac{\epsilon^{a_{1}+\beta}}{a^{a_{1}}}}\left|\delta_{\frac{1}{a}} x\right| .
$$


Therefore,

$$
\left|\delta_{\frac{1}{a}} x\right| \geq 1+\frac{\epsilon^{a_{1}+\beta}}{a^{a_{1}}} .
$$

Similarly,

$$
1 \geq\left|\delta \frac{1}{\left(b^{a_{1}}-\epsilon^{a_{1}+\beta}\right)^{1 / a_{1}}} x\right| \geq \frac{1}{1-\frac{\epsilon^{a_{1}+\beta}}{b^{a_{1}}}}\left|\delta_{\frac{1}{b}} x\right|,
$$

so that

$$
\left|\delta_{\frac{1}{b}} x\right| \leq 1-\frac{\epsilon^{a_{1}+\beta}}{b^{a_{1}}} .
$$

Now (17) and (20) tell us that

$$
\left|\delta_{\frac{1}{a}} y\right|=\left|\delta_{\frac{1}{a}} x-\delta_{\frac{1}{a}}(x-y)\right| \geq 1+\frac{\epsilon^{a_{1}+\beta}}{a^{a_{1}}}-\frac{\epsilon^{a_{1}+\beta}}{a^{a_{1}}}=1 .
$$

Also, by (18) and (21),

$$
\left|\delta_{\frac{1}{b}} y\right|=\left|\delta_{\frac{1}{b}}(y-x)+\delta_{\frac{1}{b}} x\right| \leq \frac{\epsilon^{a_{1}+\beta}}{b^{a_{1}}}+1-\frac{\epsilon^{a_{1}+\beta}}{b^{a_{1}}}=1 .
$$

Hence $a \leq \rho(y) \leq b$.

Next we construct subintervals $I_{k}$ of $(0,1]$ such that $e^{i / \rho(y)^{\beta}}$ does not oscillate rapidly when $\rho(y)^{a_{1}} \in I_{k}$.

Lemma 2. There exist two positive numbers $A_{0}$ and $B_{0}$, with $B_{0}<A_{0}^{1 / \beta}<1$, such that whenever $0<\epsilon<B_{0}$ and $1 \leq k \leq A_{0} \epsilon^{-\beta}$ ( $k$ an integer), the following hold.

(i) $4 \epsilon^{a_{1}+\beta}<\frac{1}{(2 \pi k-\pi / 3)^{a_{1} / \beta}}-\frac{1}{(2 \pi k+\pi / 3)^{a_{1} / \beta}}$ and $\epsilon \leq \frac{1}{(2 \pi k+\pi / 3)^{1 / \beta}}$.

(ii) Let

$$
I_{k}=\left[\frac{1}{(2 \pi k+\pi / 3)^{a_{1} / \beta}}+\epsilon^{a_{1}+\beta}, \frac{1}{(2 \pi k-\pi / 3)^{a_{1} / \beta}}-\epsilon^{a_{1}+\beta}\right]
$$

and

$$
J_{k}=\left[\frac{1}{(2 \pi(k+1)-\pi / 3)^{a_{1} / \beta}}-\epsilon^{a_{1}+\beta}, \frac{1}{(2 \pi k+\pi / 3)^{a_{1} / \beta}}+\epsilon^{a_{1}+\beta}\right] .
$$

Also, let $k^{\prime}$ be "the $k$ " such that $k^{\prime} \leq A_{0} \epsilon^{-\beta}<k^{\prime}+1$. Then $2 A_{0}^{-a_{1} / \beta} \epsilon^{a_{1}}<7^{-a_{1} / \beta}$ and

$$
I_{k^{\prime}} \cup\left[\bigcup_{k=1}^{k^{\prime}-1}\left(I_{k} \cup J_{k}\right)\right] \supset\left[A_{0}^{-a_{1} / \beta} \epsilon^{a_{1}}, 7^{-a_{1} / \beta}\right] .
$$

(iii) $\left|J_{k}\right| \leq C\left|I_{k+1}\right|$ for some constant $C$ that only depends on $a_{1}$ and $\beta$.

Proof. Set

$$
A_{0}=\operatorname{Min}\left[\frac{1}{4 \pi},\left(\frac{a_{1} \pi}{6 \beta(3 \pi)^{\frac{a_{1}+\beta}{\beta}}}\right)^{\frac{\beta}{a_{1}+\beta}}\right]
$$

and

$$
B_{0}=\operatorname{Min}\left[\left(\frac{1}{2}\right)^{1 / a_{1}}\left(\frac{A_{0}}{7}\right)^{1 / \beta},\left(\left(\frac{3}{5 \pi}\right)^{a_{1} / \beta}-\left(\frac{1}{7}\right)^{a_{1} / \beta}\right)^{\frac{1}{a_{1}+\beta}}\right]
$$


(i) Let $f(x)=x^{-a_{1} / \beta}(x>0)$. Then $f^{\prime}(x)=-\left(a_{1} / \beta\right) x^{-\frac{a_{1}+\beta}{\beta}}$. For $k \geq 1$,

$$
\begin{aligned}
\frac{1}{(2 \pi k-\pi / 3)^{a_{1} / \beta}}-\frac{1}{(2 \pi k+\pi / 3)^{a_{1} / \beta}} & =f(2 \pi k-\pi / 3)-f(2 \pi k+\pi / 3) \\
& =(-2 \pi / 3) f^{\prime}(t) \\
& =\frac{2 \pi a_{1}}{3 \beta} \frac{1}{t^{\frac{a_{1}+\beta}{\beta}}},
\end{aligned}
$$

where $2 \pi k-\pi / 3<t<2 \pi k+\pi / 3<3 \pi k$. Thus,

$$
\begin{aligned}
\frac{1}{(2 \pi k-\pi / 3)^{a_{1} / \beta}}-\frac{1}{(2 \pi k+\pi / 3)^{a_{1} / \beta}} & >\frac{2 \pi a_{1}}{3 \beta(3 \pi)^{\frac{a_{1}+\beta}{\beta}}} \frac{1}{k^{\frac{a_{1}+\beta}{\beta}}} \\
& \geq 4 A_{0}^{\frac{a_{1}+\beta}{\beta}} \frac{1}{k^{\frac{a_{1}+\beta}{\beta}}} .
\end{aligned}
$$

So for $1 \leq k \leq A_{0} \epsilon^{-\beta}$, we have

$$
4 \epsilon^{a_{1}+\beta}<\frac{1}{(2 \pi k-\pi / 3)^{a_{1} / \beta}}-\frac{1}{(2 \pi k+\pi / 3)^{a_{1} / \beta}} .
$$

Also, since $A_{0} \leq 1 /(4 \pi)$,

$$
\epsilon \leq \frac{A_{0}^{1 / \beta}}{k^{1 / \beta}} \leq \frac{1}{(4 \pi)^{1 / \beta}} \frac{1}{k^{1 / \beta}} \leq \frac{1}{(2 \pi k+\pi / 3)^{1 / \beta}} .
$$

(ii) By our choice of $A_{0}$ and $B_{0}$, we have

$$
2^{1 / a_{1}} A_{0}^{-1 / \beta} \epsilon<7^{-1 / \beta} \text { and } \frac{1}{(2 \pi-\pi / 3)^{a_{1} / \beta}}-\epsilon^{a_{1}+\beta}>7^{-a_{1} / \beta} .
$$

The second inequality in (22) tells us that $7^{-a_{1} / \beta} \in I_{k^{\prime}} \cup\left[\bigcup_{k=1}^{k^{\prime}-1}\left(I_{k} \cup J_{k}\right)\right]$. Now

$$
2 \pi k^{\prime}-\frac{\pi}{3}>4 k^{\prime}>k^{\prime}+1>A_{0} \epsilon^{-\beta},
$$

so that

$$
A_{0}^{-a_{1} / \beta} \epsilon^{a_{1}}>\frac{1}{\left(2 \pi k^{\prime}-\pi / 3\right)^{a_{1} / \beta}}>\frac{1}{\left(2 \pi k^{\prime}-\pi / 3\right)^{a_{1} / \beta}}-\epsilon^{a_{1}+\beta} .
$$

Thus,

$$
I_{k^{\prime}} \cup\left[\bigcup_{k=1}^{k^{\prime}-1}\left(I_{k} \cup J_{k}\right)\right] \supset\left[A_{0}^{-a_{1} / \beta} \epsilon^{a_{1}}, 7^{-a_{1} / \beta}\right] .
$$

(iii) Let $a=2 \pi k+\pi / 3$ and $d=2 \pi / 3$. Then

$$
\left|J_{k}\right|+\left|I_{k+1}\right|=f(a)-f(a+3 d)=(-3 d) f^{\prime}\left(s_{1}\right),
$$

where $a<s_{1}<a+3 d$. On the other hand,

$$
\left|I_{k+1}\right|+2 \epsilon^{a_{1}+\beta}=f(a+2 d)-f(a+3 d)=(-d) f^{\prime}\left(s_{2}\right)
$$


with $a+2 d<s_{2}<a+3 d<2 a<2 s_{1}$. Then

$$
\begin{aligned}
\left|I_{k+1}\right|+\left|J_{k}\right| & =\frac{3 d a_{1}}{\beta}\left(\frac{1}{s_{1}}\right)^{\frac{a_{1}+\beta}{\beta}} \\
& \leq \frac{3 d a_{1}}{\beta}\left(\frac{2}{s_{2}}\right)^{\frac{a_{1}+\beta}{\beta}} \\
& =3\left(2^{\frac{a_{1}+\beta}{\beta}}\right) \frac{d a_{1}}{\beta}\left(\frac{1}{s_{2}}\right)^{\frac{a_{1}+\beta}{\beta}} \\
& =3\left(2^{\frac{a_{1}+\beta}{\beta}}\right)\left(\left|I_{k+1}\right|+2 \epsilon^{a_{1}+\beta}\right) \\
& \leq 6\left(2^{\frac{a_{1}+\beta}{\beta}}\right)\left|I_{k+1}\right| .
\end{aligned}
$$

Hence

$$
\left|J_{k}\right| \leq C\left|I_{k+1}\right|
$$

This completes the proof.

Proof of Theorem 5. If $\alpha=\nu$, the right-hand side of (16) is $1 / 2$ and there is nothing to prove. So we may assume $\alpha>\nu$. Moreover, since $T$ is translation invariant, it is enough to prove the theorem for $1 \leq p \leq 2$. Let $A_{0}, B_{0}, I_{k}, J_{k}, k, k^{\prime}$, and $\epsilon$ be as in Lemma 2] Fix $\varphi \in C_{0}^{\infty}$ such that $0 \leq \varphi \leq 1, \varphi(x)=1$ for $\rho_{\beta}(x) \leq 1 / 2$, and $\varphi(x)=0$ for $\rho_{\beta}(x) \geq 1$. Define

$$
\varphi_{\epsilon}(x)=\varphi\left(\gamma_{1 / \epsilon} x\right)
$$

Then

$$
\int\left|\varphi_{\epsilon}(x)\right|^{p} d x=A_{p} \epsilon^{2 \beta+\nu}
$$

for some $A_{p}>0$.

Suppose $\rho(x)^{a_{1}} \in I_{k}$ and $\rho_{\beta}(x-y) \leq \epsilon$. Then Lemma 1, together with part (i) of Lemma 2] tell us that

$$
\frac{1}{(2 \pi k+\pi / 3)^{1 / \beta}} \leq \rho(y) \leq \frac{1}{(2 \pi k-\pi / 3)^{1 / \beta}}
$$

or

$$
2 \pi k-\pi / 3 \leq \frac{1}{\rho(y)^{\beta}} \leq 2 \pi k+\pi / 3
$$

Now by (2),$\rho(x-y) \leq \epsilon$. Also by part (i) of Lemma 2 , $\epsilon \leq \rho(x)$. Thus,

$$
\rho(y) \leq C(\rho(x-y)+\rho(x)) \leq(\epsilon+\rho(x)) \leq 2 C \rho(x)
$$


Choose $\epsilon^{\prime}$ such that $0<\epsilon^{\prime}<\epsilon$. (24) and (25)) tell us that if $\rho(x)^{a_{1}} \in I_{k}$, then

$$
\begin{aligned}
\left|\int_{1 \geq \rho(y) \geq \epsilon^{\prime}} \frac{e^{i / \rho(y)^{\beta}}}{\rho(y)^{\alpha}} \varphi_{\epsilon}(x-y) d y\right| & \geq\left|\int_{1 \geq \rho(y) \geq \epsilon^{\prime}} \frac{\cos \left(1 / \rho(y)^{\beta}\right)}{\rho(y)^{\alpha}} \varphi_{\epsilon}(x-y) d y\right| \\
& \geq \frac{1}{2} \int_{\rho_{\beta}(x-y) \leq \epsilon} \frac{1}{\rho(y)^{\alpha}} \varphi_{\epsilon}(x-y) d y \\
& \geq \frac{c}{\rho(x)^{\alpha}} \int_{\rho_{\beta}(x-y) \leq \epsilon} \varphi_{\epsilon}(x-y) d y \\
& =\frac{c}{\rho(x)^{\alpha}} \int \varphi_{\epsilon}(y) d y \\
& =\frac{c}{\rho(x)^{\alpha}} A_{1} \epsilon^{2 \beta+\nu} .
\end{aligned}
$$

Hence, if $\rho(x)^{a_{1}} \in I_{k}$,

$$
\left|T \varphi_{\epsilon}(x)\right|=\lim _{\epsilon^{\prime} \rightarrow 0}\left|\int_{1 \geq \rho(y) \geq \epsilon^{\prime}} \frac{e^{i / \rho(y)^{\beta}}}{\rho(y)^{\alpha}} \varphi_{\epsilon}(x-y) d y\right| \geq c \epsilon^{2 \beta+\nu} \frac{1}{\rho(x)^{\alpha}} .
$$

Then

$$
\begin{aligned}
\int\left|T \varphi_{\epsilon}(x)\right|^{p} d x & \geq \sum_{k} \int_{\rho(x)^{a_{1}} \in I_{k}}\left|T \varphi_{\epsilon}(x)\right|^{p} d x \\
& \geq c \epsilon^{p(2 \beta+\nu)} \sum_{k} \int_{\rho(x)^{a_{1} \in I_{k}}} \frac{d x}{\rho(x)^{\alpha p}} .
\end{aligned}
$$

Changing $\int_{\rho(x)^{a_{1} \in I_{k}}}\left(1 / \rho(x)^{\alpha p}\right) d x$ into polar coordinates, and making a simple change of variables, we get

$$
\int_{\rho(x)^{a_{1}} \in I_{k}} \frac{d x}{\rho(x)^{\alpha p}} \geq c \int_{I_{k}} \frac{d r}{r^{\left(\alpha p-a_{2}\right) / a_{1}}} .
$$

Now, using the fact that $\left|J_{k}\right| \leq C\left|I_{k+1}\right|$ (part (iii) of Lemma 21), we have

$$
\begin{aligned}
\int\left|T \varphi_{\epsilon}(x)\right|^{p} d x & \geq c \epsilon^{p(2 \beta+\nu)} \sum_{k} \int_{I_{k}} \frac{d r}{r^{\left(\alpha p-a_{2}\right) / a_{1}}} \\
& \geq c \epsilon^{p(2 \beta+\nu)}\left(\sum_{k=1}^{k^{\prime}} \int_{I_{k}} \frac{d r}{r^{\left(\alpha p-a_{2}\right) / a_{1}}}+\sum_{k=1}^{k^{\prime}-1} \int_{J_{k}} \frac{d r}{r^{\left(\alpha p-a_{2}\right) / a_{1}}}\right) \\
& \geq c \epsilon^{p(2 \beta+\nu)} \int_{I_{k^{\prime}} \cup\left[\bigcup_{k=1}^{k^{\prime}-1}\left(I_{k} \cup J_{k}\right)\right]} \frac{d r}{r^{\left(\alpha p-a_{2}\right) / a_{1}}} .
\end{aligned}
$$

Using part (ii) of Lemma 2 we get

$$
\int\left|T \varphi_{\epsilon}(x)\right|^{p} d x \geq c \epsilon^{p(2 \beta+\nu)} \int_{A_{0}^{-a_{1} / \beta} \epsilon^{a_{1}}}^{7^{-a_{1} / \beta}} \frac{d r}{r^{\left(\alpha p-a_{2}\right) / a_{1}}} .
$$

By the assumptions made on $\alpha$ and $p$ at the beginning of the proof, $\alpha p-\nu+1>1$. Hence

$$
\int\left|T \varphi_{\epsilon}(x)\right|^{p} d x \geq c \epsilon^{p(2 \beta+\nu)} \epsilon^{\nu-\alpha p} .
$$

Now, since $T$ is bounded on $L^{p}$,

$$
A_{p} \epsilon^{2 \beta+\nu}=\left\|\varphi_{\epsilon}\right\|_{L^{p}}^{p} \geq c\left\|T \varphi_{\epsilon}\right\|_{L^{p}}^{p}=c \epsilon^{p(2 \beta+\nu)} \epsilon^{\nu-\alpha p} .
$$


Letting $\epsilon \rightarrow 0$, it follows that

$$
p(2 \beta+\nu)+\nu-\alpha p \geq 2 \beta+\nu,
$$

or

$$
p(2 \beta-\alpha+\nu) \geq 2 \beta
$$

Therefore,

$$
\frac{1}{p}-\frac{1}{2} \leq \frac{\beta-\alpha+\nu}{2 \beta}
$$

This completes the proof of the theorem.

\section{REFERENCES}

1. R. R. Coifman And G. Weiss, Extensions of Hardy spaces and their use in analysis, Bull. Amer. Math. Soc. 83 (1977), 569-645. MR 56:6264

2. C. Fefferman, Inequalities for strongly singular convolution operators, Acta Math. 124 (1970), 9-36. MR 41:2468

3. C. Fefferman and E. M. Stein, $H^{p}$ spaces of several variables, Acta Math. 129 (1972), 137-193. MR 56:6263

4. A. Miyachi, On some Fourier multipliers for $H^{p}\left(\mathbb{R}^{n}\right)$, J. Fac. Sci. Univ. Tokyo 27 (1980), 157-179. MR 81g:42020

5. P. SJöLIN, $L^{p}$ estimates for strongly singular convolution operators in $\mathbb{R}^{n}$, Ark. Mat. 14 (1976), 59-64. MR 54:844

6. - , An $H^{p}$ inequality for strongly singular integrals, Mat. Zeit. 165 (1979), 231-238. MR 81d:42030

7. — Convolution with oscillating kernels, Indiana Univ. Math. J. 30 (1981), 47-56. MR 82d:42018

8. E. M. Stein, Harmonic Analysis: Real-Variable Methods, Orthogonality, and Oscillatory Integrals, Princeton Univ. Press, 1993. MR 95c:42002

9. E. M. Stein And S. Wainger, Problems in harmonic analysis related to curvature, Bull. Amer. Math. Soc. 84 (1978), 1239-1295. MR 80k:42023

10. S. Wainger, Special Trigonometric Series in $k$ Dimensions, Memoirs Amer. Math. Soc. \# 59, American Mathematical Society, Providence, RI, 1965. MR 32:320

Department of Mathematics, American University of Beirut, Beirut, Lebanon

E-mail address: bshayya@aub.edu.lb 